\title{
Skin barrier disruption by acetone: observations in a hairless mouse skin model
}

\author{
Robert Rissmann • Marion H. M. Oudshoorn • \\ Wim E. Hennink · Maria Ponec · Joke A. Bouwstra
}

Received: 24 November 2008 / Revised: 6 March 2009 / Accepted: 12 March 2009 / Published online: 7 April 2009

(C) The Author(s) 2009. This article is published with open access at Springerlink.com

\begin{abstract}
To disrupt the barrier function of the skin, different in vivo methods have been established, e.g., by acetone wiping or tape-stripping. In this study, the acetoneinduced barrier disruption of hairless mice was investigated in order to establish a reliable model to study beneficial, long-term effects on barrier recovery after topical application. For both treatments (i.e., acetone treatment and tapestripping) the transepidermal water loss directly after disruption and the subsequent barrier recovery profile were similar. Histological assessment showed significant lower number of corneocyte layers in acetone-treated and tapestripped skin compared to untreated skin, while there was no statistical difference between the two treatments. Lipid analysis of acetone-treated skin revealed that only small fraction of lipids were extracted consisting of predominantly nonpolar lipids. Importantly, the ratio of the barrier lipids, i.e., cholesterol, free fatty acids and ceramides, remained similar between control and acetone-treated skin. This reflects the undisrupted lipid organization, as determined by small-angle X-ray diffraction measurements: the long-periodicity lamellar phase was still present after acetone treatment. Our results contradict earlier studies which reported no mechanical stratum corneum removal, a substantial extraction of lipids and disruption in lipid organization. In conclusion, our studies demonstrate that barrier
\end{abstract}

R. Rissmann · M. Ponec · J. A. Bouwstra $(\bowtie)$

Department of Drug Delivery Technology,

Leiden/Amsterdam Center for Drug Research (LACDR),

Leiden University, P.O. Box 9502, 2300 RA Leiden,

The Netherlands

e-mail: Bouwstra@chem.LeidenUniv.nl

M. H. M. Oudshoorn · W. E. Hennink

Department of Pharmaceutics,

Utrecht Institute for Pharmaceutical Sciences (UIPS),

Utrecht University, Utrecht, The Netherlands disruption due to acetone treatment is mainly due to removal of corneocytes.

Keywords Lipid organization - Skin barrier perturbation . Murine model
Abbreviations
HPTLC High-performance thin-layer chromatography
LPP Long periodicity phase
SAXD Small-angle X-ray diffraction
SC Stratum corneum
TEWL Transepidermal water loss
VC Vernix caseosa

\section{Introduction}

The uppermost layer of the epidermis, the stratum corneum (SC), comprises the main barrier function of the skin. To reduce the skin barrier function different in vivo models have been established. Commonly used laboratory techniques are removal of SC cell layers by sequential tapestripping and wiping the SC surface with organic solvents such as acetone [7, 9, 10, 19]. Typically, these studies are combined with monitoring the skin barrier (recovery) by measuring the transepidermal water loss (TEWL). For more fundamental insights of the mechanism of the barrier perturbation, additional methods to TEWL measurements were applied: (1) analysis of the SC lipids to monitor the lipid extraction [6,8], (2) fluorescence microscopy to visualize the remaining lipophilic regions in the SC [8] and (3) electron microscopy with $\mathrm{RuO}_{4}$ post-fixation to visualize the lipid organization in the SC [18].

Our general aim is to establish a reliable barrier disruption method to study the long-term effects on barrier recovery 
after topical application of pharmaceuticals. In a previous study, we thoroughly investigated different disruption levels by tape-stripping [12]. In most cases a very fast recovery (i.e., 3 days), as monitored by TEWL, was observed. Only when the highest degree of barrier disruption was applied, in which the SC was completely removed, it took approximately 8 days to completely restore the skin barrier function [12]. Aim of the present study was to evaluate the acetone-induced barrier disruption barrier repair. Barrier disruption and lipid extraction of hairless mouse skin was achieved by wiping with acetone-soaked swabs as described previously $[7,8,10,18]$. After disruption, we examined the degree of skin barrier disruption (by TEWL), lipid composition (by high-performance thin-layer chromatography, HPTLC), lipid organization (by small-angle Xray diffraction, SAXD) and the morphology (confocal laser scanning microscopy and light microscopy). As comparison, the morphological features and TEWL of tape-stripped skin were also explored.

\section{Materials and methods}

\section{Skin barrier disruption}

Male hairless mice (SKH1-hr, Charles River, St-Aubin-lesElbeuf, France) were 7-9 weeks old and $28 \pm 2 \mathrm{~g}$ in weight. All animal experiments had been approved by the Research Ethical Committee of Leiden University. Prior to skin barrier disruption, the back of the mice was carefully rinsed with water-soaked tissues. No changes in TEWL values were observed after washing. As shown in Table 1, after approximately $11 \mathrm{~min}$ of acetone treatment with cotton swabs, during which one flank of the back was treated with approximately ten acetone wipes, a TEWL of $\sim 60 \mathrm{~g} / \mathrm{m}^{2} / \mathrm{h}$ was obtained. One wipe consisted of moving a delipidized, acetone-soaked cotton swab four times back and forth over the skin surface. The contralateral flank was left untreated. As reference for barrier disruption, sequential tape-stripping (black D-squame tape, Cuderm, Dallas, USA) in alternating direction of the stretched skin $[12,13]$ was performed until a TEWL of $\sim 60 \mathrm{~g} / \mathrm{m}^{2} / \mathrm{h}$ was reached (seven strips). The level of barrier disruption was assessed by measuring the TEWL using a Tewameter TM 210 (Courage \& Khazaka, Cologne, Germany). Skin biopsies were taken directly after disruption from the sacrificed animals.

\section{Histology}

Histological assessment was performed as described previously [12, 13]. In brief, $5 \mu \mathrm{m}$ thick cryostat sections of the biopsies were stained with $1 \%$ safranin solution and subsequently treated with $2 \% \mathrm{KOH}$ solution to expand the SC
Table 1 Time protocol of acetone-induced skin barrier disruption

\begin{tabular}{lc}
\hline Time $(\mathrm{min})$ & TEWL $\left(\mathrm{g} / \mathrm{h} / \mathrm{m}^{2}\right)$ \\
\hline 0 & 9.4 \\
3.5 & 9.8 \\
5.5 & 13.5 \\
7 & 25.9 \\
9.5 & 47.3 \\
$>11$ & $>60$ \\
\hline
\end{tabular}

The skin barrier is disrupted by means of acetone-soaked cotton swabs during various time periods. The barrier disruption is monitored by measuring the transepidermal water loss (TEWL)

[17]. Digital pictures of the light-microphotographs were captured using a Carl Zeiss axioskop (Jena, Germany) and the average of the SC layers was determined from 20 random locations of the cross-sections. Statistical differences between the groups (i.e., untreated, acetone-treated and tape-stripped) were determined by an one-way ANOVA with a Bonferroni post-test.

In order to visualize the lipid matrix of the cross-sections staining with Nile Red was performed. Prior to addition of a droplet Nile Red solution [2.5 $\mu \mathrm{g} / \mathrm{ml}(\mathrm{w} / \mathrm{v})$ in acetone] on the frozen sections [8], the SC was expanded (see above) and visualized by confocal laser scanning microscopy on a Bio-Rad Radiance 2100 system with a Nikon Eclipse TE2000-U (Nikon, Japan) inverted microscope.

Analysis of lipid composition and organization

For lipid analysis, the epidermis of the biopsies $(n=4)$ was heat separated from the underlying tissue. In brief, the biopsies were sandwiched between two preheated cover slips with the SC side facing downward. After 2 min equilibration at $60^{\circ} \mathrm{C}$, the epidermis was carefully peeled of by means of tweezers. Extraction of the lipids was performed sequentially by different mixtures of chloroform/methanol according to a modified protocol of Bligh and Dyer [1]. Lipid composition was determined by HPTLC as described previously [14].

In order to determine the lipid organization, SC sheets of the biopsies $(n=3)$ were obtained after overnight trypsin incubation [16]. The SC sheets were then measured by SAXD at the European Synchrotron Radiation Facility in Grenoble, France. A more detailed description of the experimental set up has been given elsewhere $[2,16]$.

\section{Results and discussion}

Untreated hairless mouse skin exhibited a TEWL of $9.7 \pm 1.5 \mathrm{~g} / \mathrm{h} / \mathrm{m}^{2}$ whereas the TEWL after acetone treat- 
Fig. 1 Different disruption methods cause abrasion of SC layers. Photomicrographs of frozen cross-sections of hairless mouse skin before (a), directly after disruption with acetone (b) and tape-stripping (c) are shown. The lipophilic fluorescent dye Nile Red stains for nonpolar lipids. The $\mathrm{KOH}$ expanded corneocytes can easily be distinguished. Alkali expanded safranin stained cross-sections of mouse epidermis are depicted in panels $\mathbf{d}$ (untreated), e (acetone-treated) and $\mathbf{f}$ (tapestripped). The corneocyte layers on 20 different locations were counted and the mean $+\mathrm{SD}$ is presented in panel $\mathbf{g}$
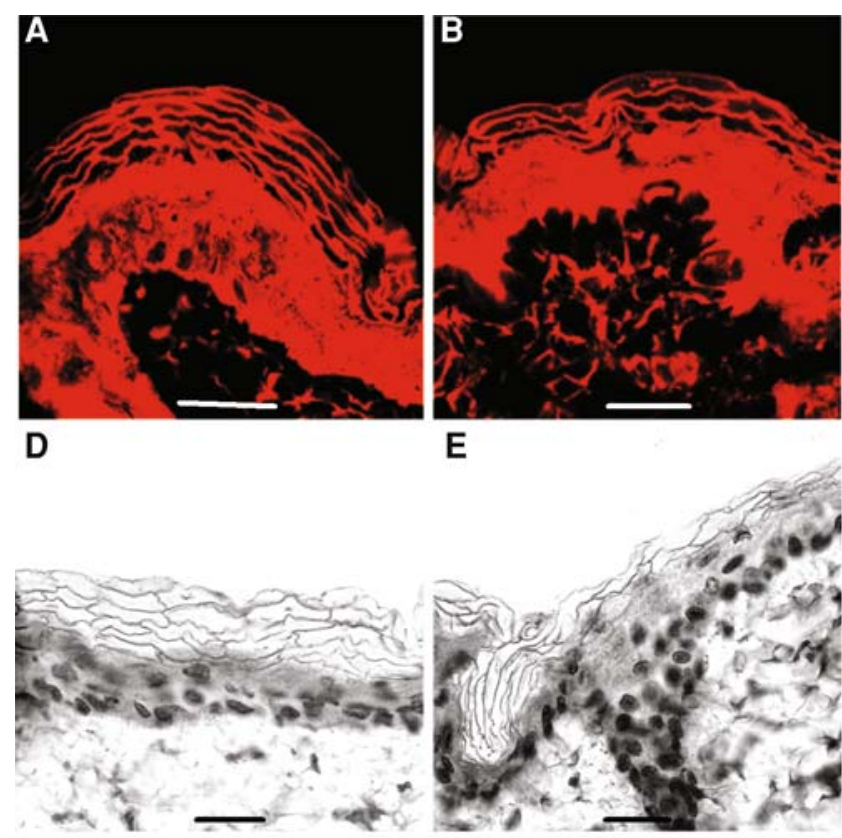

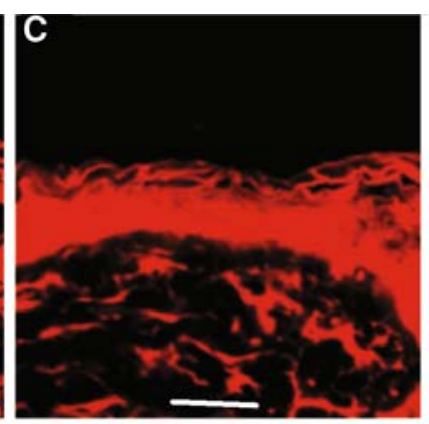

$\mathbf{F}$
E

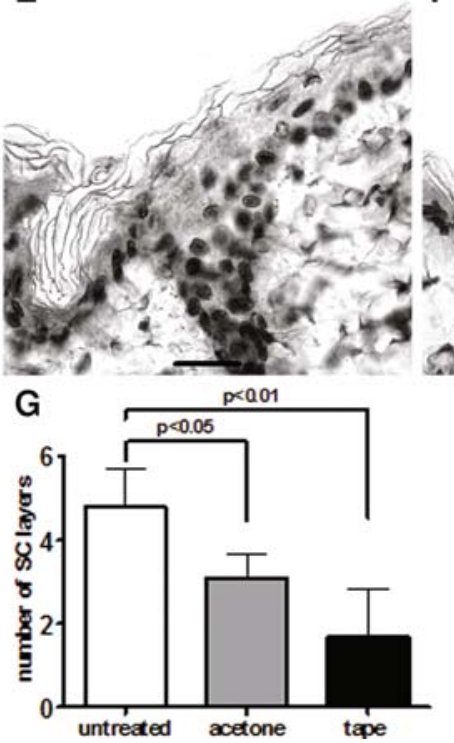

ment was $65 \pm 10 \mathrm{~g} / \mathrm{h} / \mathrm{m}^{2}$. In order to compare the two disruption methods, a similar barrier disruption was achieved by sequential tape-stripping (TEWL of $66 \pm 3 \mathrm{~g} / \mathrm{h} / \mathrm{m}^{2}$ ). The TEWL measurements of the barrier recovery showed similar profiles for both disruption methods and complete recovery was observed 3 days post-disruption (not shown). The histological features of Nile Red stained cross-sections of the skin, obtained directly after disruption, are depicted in Fig. 1 and compared to untreated skin. Untreated skin clearly showed the presence of the lipophilic dye between the alkali expanded corneocytes (Fig. 1a). Interestingly, a lower number of SC layers was discernable directly after acetone treatment. However, no changes in the pattern or intensity of the Nile Red staining were observed, indicating that no alteration in the lipid distribution within the SC occurred (Fig. 1b). The light microscopic observations confirmed the reduced amount of corneocyte layers after acetone treatment (Fig. 1e) compared to untreated (Fig. 1d). Tape-stripped skin (Fig. 1c, f) also exhibited a lower number of SC layers than untreated skin (Fig. 1a, d) while the lipid distribution was maintained. When quantifying the SC layers of both disruption methods, a similar trend was observed in the photomicrographs: a significant reduced number of SC layers between untreated and both disruption methods could be detected (Fig. 1g). This indicates that during the gentle acetone-wipe a significant amount of corneocytes was removed, which accounts for a substantial barrier perturbation. The cross-sections of tape-stripped skin showed the trend to a more pronounced reduction of the SC thickness compared to acetone treatment, however no significant difference between both treatments was determined.

After acetone treatment, the lipid composition in the applied swab and the remaining epidermis was explored. Lipid analysis of tape-stripped skin was not possible, as the remaining tissue was too fragile for processing. The lipid analysis of the acetone-treated epidermis by HPTLC is shown in Fig. 2a. The lipid extracts of the untreated (lane 1) and acetone-treated epidermis (lane 2) showed very similar lipid distribution: the nonpolar skin surface lipids, i.e., sterol esters and triglycerides, are the predominant lipid classes. Some lipids were removed by acetone as the cotton swab extract consists mainly of nonpolar lipids (lane 3 ). Around $30 \%$ of the total lipids (w/w) were extracted as determined gravimetrically. Importantly, no changes in the composition of the barrier lipids, cholesterol, free fatty acids and ceramides could be detected by the HPTLC technique when comparing acetone-treated compared to untreated epidermis (comparison of lanes 1 and 2). This observation contrasts previous studies reporting that barrier 


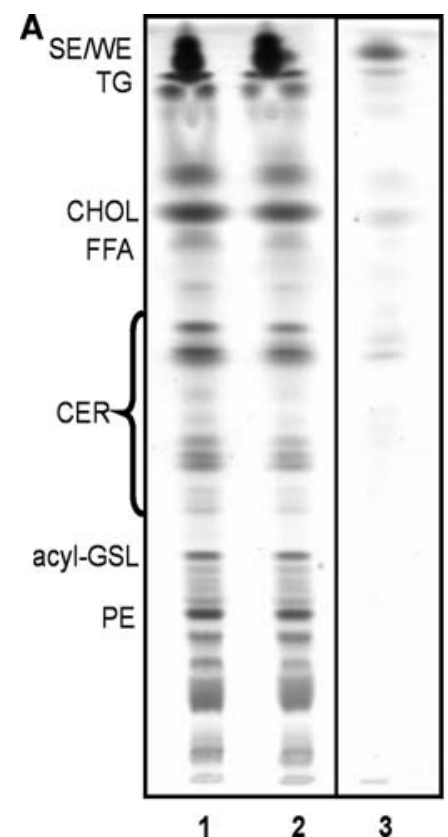

Fig. 2 Only little changes in lipid composition and organization after barrier disruption with acetone. A HPTLC of lipid extracts from untreated mouse epidermis (lane 1), acetone-treated epidermis (lane 2) and lipids extracted by the acetone swab (lane 3 ) are depicted in panel a. The amount of lipids applied per lane, represent a skin surface of $10 \mathrm{~mm}^{2}$. Legend on the left indicates the $R_{\mathrm{f}}$-values of different compounds. $S E / W E$ sterol esters/wax esters, $T G$ triglycerides, $C H O L$ cholesterol, FFA free fatty acids, CER ceramides, acyl-GSL acyl-

lipids were extracted by acetone-soaked cotton balls in substantial amounts $[6,8]$. This is of major importance as the barrier lipids play a crucial role for the SC permeability barrier $[3,4]$. Now, the question rises whether the SC lipid organization was disturbed. The results obtained with SAXD on isolated mouse SC are depicted in Fig. 2b. The upper two patterns (i.e., control and acetone-treated site) showed similar diffraction patterns: a very weak first $(q=0.46 / \mathrm{nm})$ and a clear second $(q=0.94 / \mathrm{nm})$ and third order $(q=1.42 / \mathrm{nm})$ of the long periodicity phase (LPP) with a repeat distance of $13.4 \mathrm{~nm}$ were discernable. The higher order reflections clearly indicated the presence of the LPP with and without acetone treatment. Therefore, a very similar lipid organization was observed prior to and after acetone treatment. This is in agreement with the presence of the remaining barrier lipids in a similar composition after acetone treatment because the lipid composition is the determining factor for the lipid phase behavior. However, the increased TEWL clearly indicates a perturbed barrier that can not be explained by the absence of the characteristic lamellar phase or by lipid extraction. Evidently, it might be attributed to a large extent to the removal of corneocytes. Compared to tape-stripped skin, the acetone-treated site showed a similar TEWL value and also a (slightly) reduced amount of SC layers (Fig. 1g). The residual loss in the barrier property was presumably due to a change in the glycosphingolipids, $P E$ phosphoethanolamine. The scattered intensity (arbitrary units) is plotted as function of scattering vector $(q)$ in panel b. SC sheets of two donors (upper two and lower two patterns) before (untreated) and after acetone treatment were directly mounted into the $\mathrm{X}$-ray beam. The roman numerals indicate the various orders of the LPP: first order (I) located at $q=0.46 / \mathrm{nm}(d=13.7 \mathrm{~nm})$, second order (II) at $q=0.94 / \mathrm{nm}(d=6.7 \mathrm{~nm})$ and third order $(I I I)$ at $q=1.42 / \mathrm{nm}$ $(d=4.4 \mathrm{~nm})$

lipids: during wiping, (barrier) lipids partially dissolve in acetone. As acetone evaporates rapidly, the dissolved lipids may rearrange by re-crystallization of the (barrier) lipids and lead to a possible formation of the LPP. The increased intensity of the first order (Fig. 2b, lowest pattern) could also be explained by this reformation of the LPP. We already demonstrated previously that the LPP can be formed after evaporation of solvents with distinct sample history, i.e., shear stresses and temperature [15].

In comparison with previous studies, the following differences were found concerning acetone treatment: (1) Virtually all Nile Red staining from the nonpolar lipids of SC was reported to be removed [8], while our confocal images showed a homogeneous distribution of this fluorescent probe within the SC (Fig. 1a, b). (2) No removal of SC layers up to a TEWL of $40 \mathrm{~g} / \mathrm{m}^{2} / \mathrm{h}$ was observed $[11,18]$, whereas we detected a significant reduced amount of SC layers at a TEWL of $60 \mathrm{~g} / \mathrm{m}^{2} / \mathrm{h}$ (Fig. 1g). (3) The removal of the bulk lipids from the SC was shown to correlate directly with the degree of barrier perturbation $[6,8]$, whereas in this study only little extraction of the lipids was observed (Fig. 2a). (4) A decrease in intercellular lamellae and consequently a lipid disorganization was reported [18], while we could not detect changes in lipid organization by SAXD, which is a bulk method (Fig. 2b). The difference in the outcome can hardly be due to the barrier disruption 
method, as only a slight variation exists in the application method (differences in cotton swabs and cotton balls). However, the different results show that a clear definition of 'gently wiping' is of importance since also the mechanical wiping has influence on the skin barrier as there is a risk of removing corneocytes.

When comparing the acetone-induced barrier disruption with tape-stripping, as methods for studying long-term effects after topical application of pharmaceuticals, the tape-stripping offers several advantages: (1) A clear correlation between cells removed and increased TEWL could be observed and a long-term recovery can be achieved [12]. (2) The number of tape-strips can be defined beforehand, which is advantageous for the degree of barrier disruption under practical conditions. (3) Although different levels of acetone-induced barrier perturbation have been established [8], no long-term recovery was reported.

Although the acetone-induced barrier disruption might not be the most appropriate method to study barrier recovery after topical application, its suitability for other mechanistic investigations, e.g., the epidermal lipid synthesis during barrier homeostasis [5] or the response of lamellar bodies upon barrier disruption [10] is indisputable. However, this short communication provides discussion points, which need to be considered when using the acetoneinduced barrier disruption method. We found evidence that the loss in barrier function is mainly due to the removal of corneocytes, whereas changes in lipid composition and organization only play a minor role. Importantly, the ratio of the barrier lipids remained similar between control and acetone-treated skin. This provides also a basis for the explanation of the undisrupted lipid organization after acetone treatment. However, our study strongly indicates that due to evaporation of the solvent, barrier lipids might recrystallize in the same structure, i.e., the LPP, as prior to the acetone treatment.

In conclusion, acetone treatment predominantly extracts nonpolar lipids, while the ratio of barrier lipids and the lipid organization remain similar. The removal of the corneocyte layers mainly accounts for the barrier disruption as was also observed for tape-stripping. In future, the tape-stripping method will be employed to investigate beneficial effects of topical applications on barrier recovery.

Acknowledgments We like to thank Dr Wim Bras and Dr Kristina Kvashnina at the European Synchrotron Radiation Facility in Grenoble, France for their valuable support. This work was financially supported by the Dutch Technology Foundation STW, grant no. LGT 6117.

Open Access This article is distributed under the terms of the Creative Commons Attribution Noncommercial License which permits any noncommercial use, distribution, and reproduction in any medium, provided the original author(s) and source are credited.

\section{References}

1. Bligh EG, Dyer WJ (1959) A rapid method of total lipid extraction and purification. Can J Biochem Physiol 37:911-917

2. Bras W (1998) A SAXS/WAXS beamline at the ESRF and future experiments. J Macromol Sci B Phys 37:557-566. doi:10.1080/ 00222349808220492

3. Elias PM (1983) Epidermal lipids, barrier function, and desquamation. J Invest Dermatol 80:S44-S49. doi:10.1111/1523-1747. ep12537108

4. Elias PM (1981) Lipids and the epidermal permeability barrier. Arch Dermatol Res 270:95-117. doi:10.1007/BF00417155

5. Feingold KR (1991) The regulation and role of epidermal lipid synthesis. Adv Lipid Res 24:57-82

6. Grubauer G, Elias PM, Feingold KR (1989) Transepidermal water loss: the signal for recovery of barrier structure and function. J Lipid Res 30:323-333

7. Grubauer G, Feingold KR, Elias PM (1987) Relationship of epidermal lipogenesis to cutaneous barrier function. J Lipid Res 28:746-752

8. Grubauer G, Feingold KR, Harris RM, Elias PM (1989) Lipid content and lipid type as determinants of the epidermal permeability barrier. J Lipid Res 30:89-96

9. Holleran WM, Takagi Y, Menon GK, Jackson SM, Lee JM, Feingold KR, Elias PM (1994) Permeability barrier requirements regulate epidermal beta-glucocerebrosidase. J Lipid Res 35:905-912

10. Menon GK, Feingold KR, Elias PM (1992) Lamellar body secretory response to barrier disruption. J Invest Dermatol 98:279-289. doi:10.1111/1523-1747.ep12497866

11. Menon GK, Feingold KR, Moser AH, Brown BE, Elias PM (1985) De novo sterologenesis in the skin. II. Regulation by cutaneous barrier requirements. J Lipid Res 26:418-427

12. Oudshoorn MHM, Rissmann R, van der Coelen D, Hennink WE, Ponec M, Bouwstra JA (2009) Development of a murine model to evaluate the effect of vernix caseosa on skin barrier recovery. Exp Dermatol 18(2):178-184. doi:10.1111/j.1600-0625.2008.00780.x

13. Oudshoorn MHM, Rissmann R, van der Coelen D, Hennink WE, Ponec M, Bouwstra JA (2009) Effect of synthetic vernix biofilms on barrier recovery of damaged mice skin. Exp Dermatol (in press)

14. Ponec M, Weerheim A, Kempenaar J, Mulder A, Gooris GS, Bouwstra JA, Mommaas MA (1997) The formation of competent barrier lipids in reconstructed human epidermis requires the presence of vitamin C. J Invest Dermatol 109:348-355. doi:10.1111/ 1523-1747.ep12336024

15. Rissmann R, Gooris GS, Ponec M, Bouwstra JA (2009) Long periodicity phase in extracted lipids of vernix caseosa obtained with equilibration at physiological temperature. Chem Phys Lipids 158(1):32-38. doi:10.1016/j.chemphyslip.2008.10.001

16. Rissmann R, Groenink HW, Weerheim AM, Hoath SB, Ponec M, Bouwstra JA (2006) New insights into ultrastructure, lipid composition and organization of vernix caseosa. J Invest Dermatol 126:1823-1833. doi:10.1038/sj.jid.5700305

17. Sakai S, Endo Y, Ozawa N, Sugawara T, Kusaka A, Sayo T, Tagami H, Inoue S (2003) Characteristics of the epidermis and stratum corneum of hairless mice with experimentally induced diabetes mellitus. J Invest Dermatol 120(1):79-85. doi:10.1046/ j.1523-1747.2003.12006.x

18. Tsai JC, Sheu HM, Hung PL, Cheng CL (2001) Effect of barrier disruption by acetone treatment on the permeability of compounds with various lipophilicities: implications for the permeability of compromised skin. J Pharm Sci 90:1242-1254. doi:10.1002/jps.1077

19. Yang L, Mao-Qiang M, Taljebini M, Elias PM, Feingold KR (1995) Topical stratum corneum lipids accelerate barrier repair after tape stripping, solvent treatment and some but not all types of detergent treatment. Br J Dermatol 133:679-685 